\title{
ALTERAÇÃO DA COR NO AÇO ASTM F138 POR MEIO DE IRRADIAÇÃO COM LASER DE FEMTOSSEGUNDO*
}

\author{
Leandro Gusmão da Silva ${ }^{1}$ \\ Ricardo Elgul Samad² \\ Nilson Dias Vieira $\mathrm{Jr}^{3}$ \\ Wagner de Rossi ${ }^{4}$
}

\section{Resumo}

Neste trabalho, foram estudados os efeitos da irradiação de uma superfície polida do aço inoxidável tipo ASTM F138 com laser de pulsos ultracurtos. As irradiações foram realizadas variando-se a velocidade de varredura do laser, ou seja, a taxa de sobreposição espacial de pulsos. Foi observado que após a irradiação houve uma visível mudança na coloração da amostra. Também foi verificado que com 0 aumento da taxa de sobreposição de pulsos a superfície tendeu a tornar-se preta, ou seja, absorvedora de luz. Com essa técnica, pode-se realizar marcações com alto contraste sem que haja formação de camadas de óxidos, nitretos ou alterações microestruturais no metal, o que é importante para um material com a precisão que um implante exige.

Palavras-chave: Femtossegundo; Cor em metais; Laser; LIPSS.

\section{COLOR CHANGE IN METALS BY MEANS OF REGULAR NANOSTRUCTURES PRODUCED BY FEMTOSECOND LASER PULSES}

\begin{abstract}
In this work, we studied the ultra-short laser pulses irradiation effects in a ASTM F138 stainless steel sample. The irradiations were performed varying the laser scanning speed, in other words, the number of overlapped laser shots. It was observed that after irradiation there was a visible change in coloration of the samples, which became darker as we increase the overlap rate. Employing this technique is a considerable way to produce high contrast markings without any changes in the metal microstructure, or the formation of coatings of oxides or nitrides which is important for a material with the level of accuracy required by this kind of implant.
\end{abstract}

Keywords: Femtosecond; Color; Laser; LIPSS.

1 Tecnólogo em Materiais, mestrando do programa de pós-graduação da universidade de São Paulo pelo Instituto de Pesquisas Energéticas e Nucleares - IPEN.

2 Bacharel em Física, Mestre e Doutor em física do estado sólido, pesquisador do centro de lasers e aplicações do Instituto de Pesquisas Energéticas e Nucleares - IPEN.

3 Bacharel em Física, Mestre em física nuclear e Doutor em Física da matéria condensada, pesquisador do centro de lasers e aplicações do Instituto de Pesquisas Energéticas e Nucleares IPEN.

4 Bacharel em Física, Mestre e Doutor em Física, pesquisador do centro de lasers e aplicações do Instituto de Pesquisas Energéticas e Nucleares - IPEN. 


\section{INTRODUÇÃO}

Há pelo menos quarenta anos [1], os danos periódicos e regulares causados por laser em sólidos vêm sendo estudados, tanto em suas aplicações, como na descoberta de uma teoria física por trás do fenômeno. Esse interesse aumentou na última década com a utilização de lasers de pulsos ultracurtos, que trouxeram para a escala micro/nanométrica tais efeitos na superfície dos materiais que são irradiados com esse tipo de laser [2].

Esses danos periódicos superficiais são chamados de Estruturas Superficiais Induzidas por Laser (Laser Induced Periodic Surface Structures), ou simplesmente LIPSS ou ripples. Podem ser, dependendo da energia aplicada no material, paralelos ou perpendiculares à direção de polarização do feixe e possuem períodos aproximadamente iguais ao comprimento de onda do laser. São criados com fluências abaixo e próximas do limiar de ablação do material, seja ele um metal [3] ou dielétrico (4).

Existem dois métodos de mudança de cor de metais por meio de tratamento a laser. O primeiro utiliza o laser como uma fonte de calor que provoca oxidação da superfície metálica, esse óxido pode assumir um comportamento de filme fino, ou seja, a luz refletida pela superfície do filme fino sofre interferência com a luz refletida pela interface do filme com o substrato-causando o efeito de mudança de cor. $\mathrm{Na}$ segunda técnica, o laser é utilizado para a fabricação de ranhuras periódicas que funcionam como uma grade de difração. A luz incidente sobre as ranhuras sofrem refração, dispersando seus diversos componentes de comprimento de onda em função do ângulo relativo ao ângulo de incidência.

Com a utilização de lasers de pulsos ultracurtos, tornou-se possível a fabricação dessas ranhuras em uma escala nanométricas, ou seja, os LIPSS, com profundidade de $100 \mathrm{~nm}$ (5) e cp, a grade vantagem de que a produção dos LIPSS ocorre por um processo ondulatório, não havendo aquecimento do material e, portanto, preservando suas propriedades microestruturais.

A aplicação de processos com laser de pulsos ultracurtos nesse e em outros materiais metálicos é justificada pelo baixo choque térmico que esse processo proporciona. Enquanto lasers com pulsos de duração na faixa de picossegundos aquecem o material localmente, levando a mudanças de fase e até mesmo à fusão pontual do material, os lasers que operam com pulsos em femtossegundo possuem uma dinâmica térmica fora de equilíbrio (7), o que proporciona um processo com altíssima energia com aquecimento praticamente nulo, impedindo mudanças de fase, choque térmico e oxidação da região irradiada.

As primeiras utilizações dos LIPSS com o intuito de mudança de cor em metais foi realizado por Vorobyev et al. (6). Os autores obtiveram várias cores em alumínio, deixando-o inclusive com aparência dourada.

Neste trabalho, foi escolhido o aço inoxidável grau F138 para que sejam estudados os efeitos ópticos da produção de LIPSS em sua superfície. A escolha desse material levou em consideração sua grande variedade de aplicações, desde a indústria aeroespacial até implantes ósseos.

\section{MATERIAIS E MÉTODOS}

Os experimentos para a produção de LIPSS por meio de laser com pulsos ultracurtos foram conduzidos através de um sistema laser de Ti:Safira. O sistema emite pulsos com 30 fs de duração, com comprimento de onda central de $800 \mathrm{~nm}$ e 
taxa de repetição de $4 \mathrm{kHz}$. A focalização do feixe se deu através de uma lente de $50 \mathrm{~mm}$ de comprimento focal, e foi utilizada energia de $1 \mu \mathrm{J}$ por pulso. $\mathrm{O}$ aço $\mathrm{F} 138$ utilizado foi obtido através de uma barra laminada com $30 \mathrm{~mm}$ de diâmetro, da qual foi cortada uma seção transversal com $10 \mathrm{~mm}$ de espessura; sua superfície foi lixada e polida. O aço F138 polido foi acoplado a um sistema motorizado, programável através de comandos $\mathrm{CNC}$, com resolução de $0,001 \mathrm{~mm}$. Os experimentos foram realizados criando-se retângulos de $1 \mathrm{~mm} \times 5 \mathrm{~mm}$ variando a velocidade de deslocamento do laser entre os retângulos, ou seja, alterando a taxa de sobreposição de pulsos

Após a fabricação das áreas irradiadas, o aço foi caracterizado com o intuito de se observar a presença e as características dos LIPSS. Para isso, foi utilizado um Microscópio Eletrônico de Varredura Hitashi Tabletop TM3000.

Para a caracterização das características ópticas foi utilizado uma câmera fotográfica convencional para o registro visual em ampliação real. Além disso, os padrões de reflexão da amostra após a incidência do laser foram obtidos através do espectrômetro Thorlabs CCS100 e do espectrofotômetro Agilent Cary 5000.

\section{RESULTADOS E DISCUSSÃO}

\subsection{Alteração da cor}

Após a incidência do feixe laser no aço, notaram-se alterações de sua coloração em função do ângulo de incidência da luz e o ângulo de visualização da amostra (Figura 1). Pode-se notar que as cores variam conforme o ângulo de incidência da luz e com o ângulo de visualização que no caso é o ângulo da câmera fotográfica em função da amostra. Além disso, nas condições em que a velocidade de propagação do feixe é menor (isto é, maior taxa de sobreposição de pulsos) a amostra tende a escurecer, chegando a uma tonalidade preta.

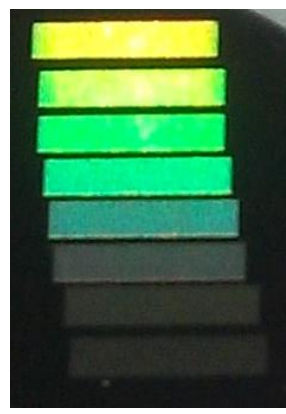

Figura 1. Retângulos coloridos sobre a superfície do aço F138 produzidos pela formação de LIPSS. O parâmetro de processo variado foi a sobreposição $\mathrm{N}$ de pulsos, de 2,5 a 96, de cima para baixo. A imagem foi obtida com a câmera posicionada em um ângulo de $45^{\circ}$ da superfície da amostra.

\subsection{Características dos LIPSS}

Com o intuito de entender o que causou essas mudanças na coloração do material, o mesmo foi analisado sobre altas ampliações nas regiões irradiadas (Figura 2 e 3). Em todas elas, foram observadas as presenças de LIPSS. Pode-se notar que em 
maiores taxas de sobreposição, os LIPSS tendem a perder a linearidade com tendência a formação de regiões granulares (seta na figura 2).

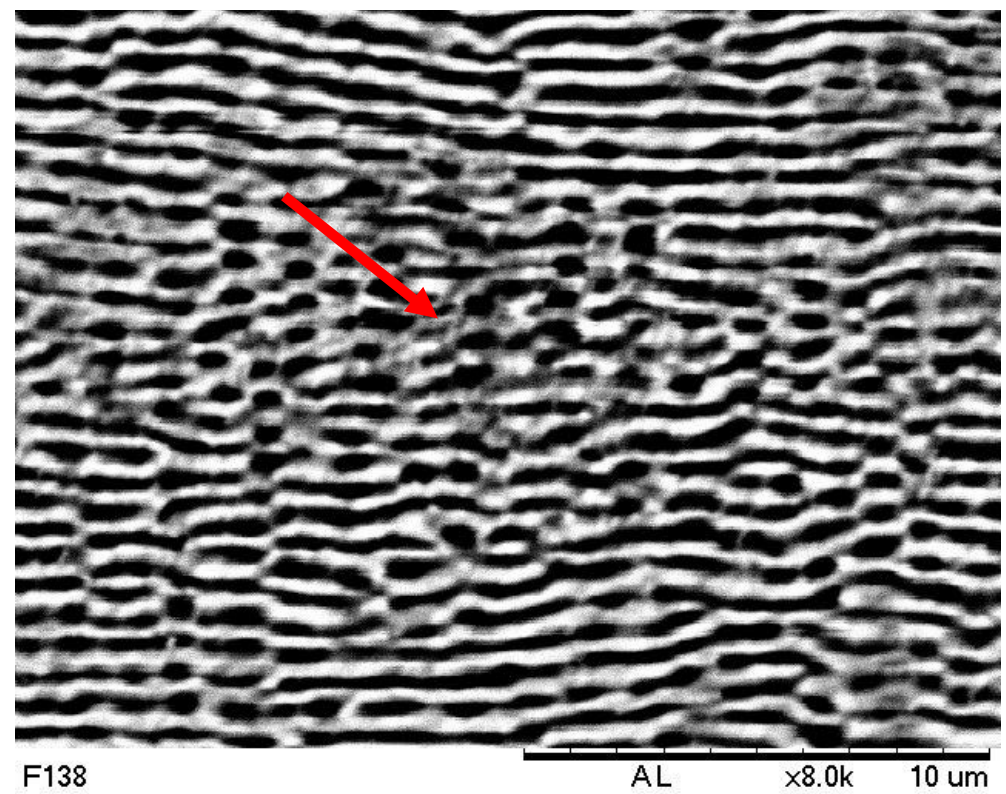

Figura 2. LIPPS produzidos com sobreposição $\mathrm{N}$ de 2,5 pulsos laser

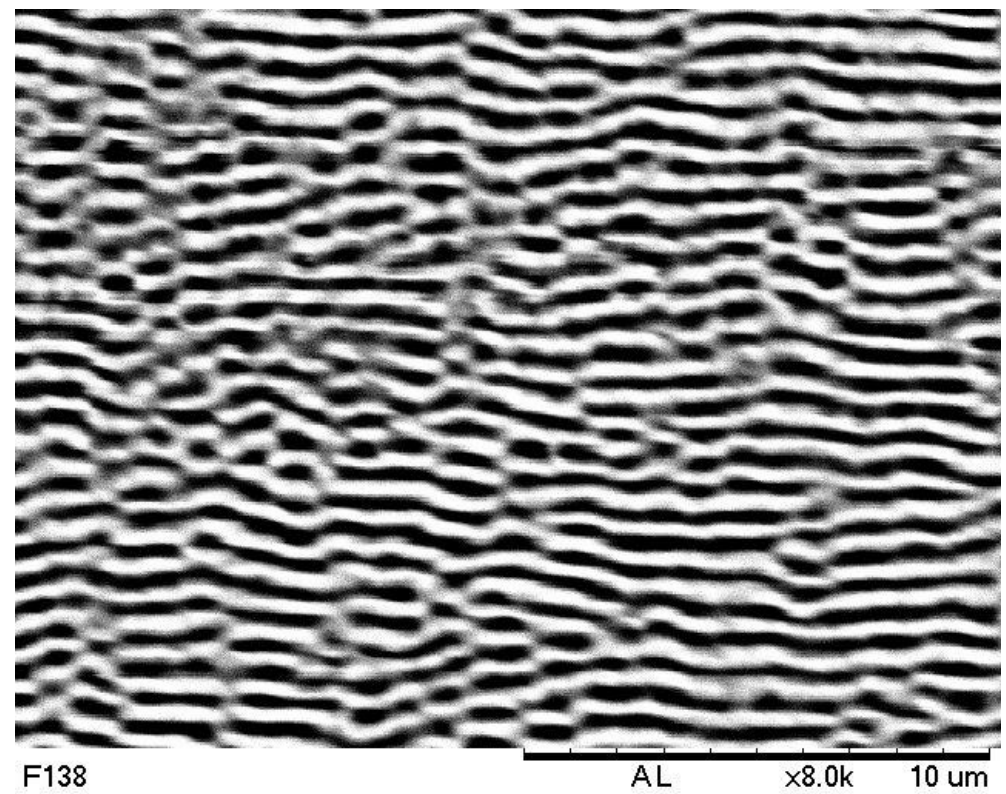

Figura 3. LIPSS produzidos com $\mathrm{N}=2,5$ pulsos laser.

Esse efeito já foi visualizado por Oliveira et al (8) que demonstraram que os LIPSS tendem a assumir a forma colunar com o aumento da energia e da taxa de sobreposição de pulsos, o que justifica a ausência do efeito de mudança de cor nas condições com altas taxas de sobreposição de pulsos.

Para entender o fenômeno de alteração da cor (característica da reflexão da luz), podem-se tratar os LIPSS como sendo uma grade de difração retangular e com isso aplicar a teoria da difração em grades periódicas. Aplicando tal teoria temos que o 
comprimento de onda $\lambda$ refletido que causa o efeito visual de mudança de cor pode ser calculado seguindo a equação de difração (Equação 1).

$$
\boldsymbol{m} \lambda=d(\sin \alpha \cdot \sin \varphi+\sin \beta) \quad \text { Equação } 1 \text { (5) }
$$

Onde $m$ é a ordem de difração, que é um número inteiro. $\lambda$ é o comprimento de onda refletido, que no espectro visível varia entre $400 \mathrm{~nm}$ e $700 \mathrm{~nm}$. $d$ é o período dos LIPSS e a função $(\sin \alpha \cdot \sin \varphi+\sin \beta)$ é resultado dos ângulos de incidência, reflexão e direção dos LIPSS, conforme figura 4.

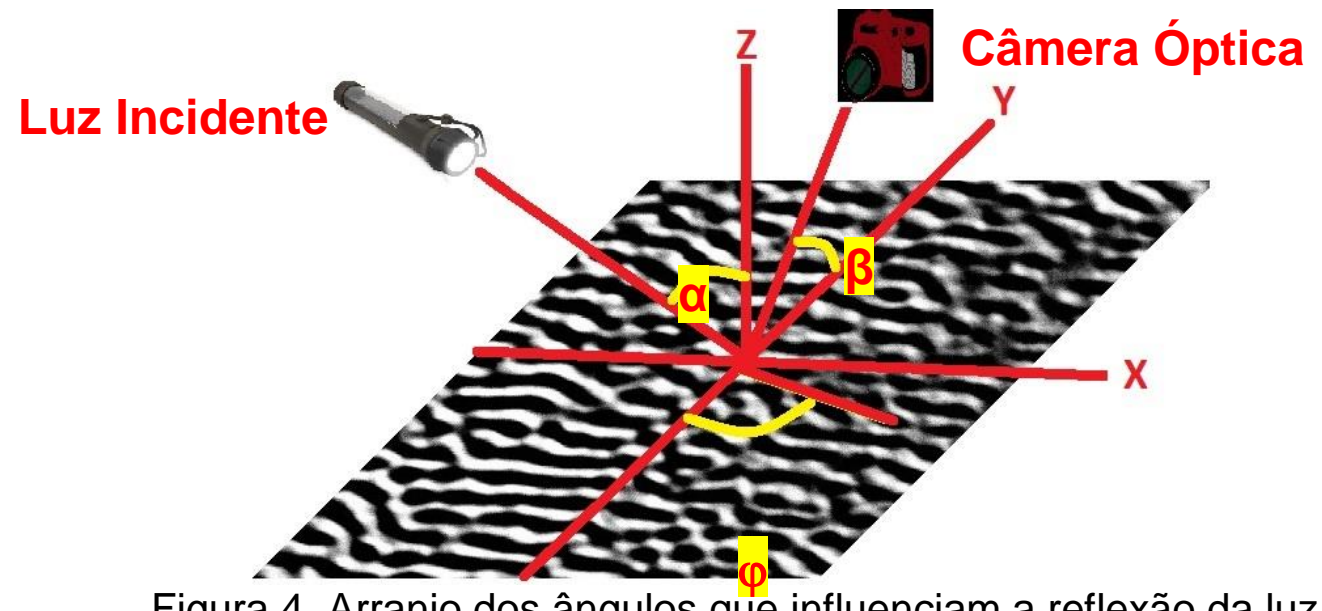

Figura 4. Arranjo dos ângulos que influenciam a reflexão da luz

A figura 1 foi obtida posicionando-se a câmera em um ângulo de $45^{\circ}$ da amostra, podemos considerar que a luz natural incidente atinge a amostra perpendicularmente à superfície e os LIPSS estão alinhados longitudinalmente aos retângulos irradiados. Aplicando esses ângulos na Equação 1, chegamos à um comprimento de onda de reflexão de aproximadamente $530 \mathrm{~nm}$, o que é coerente com a coloração esverdeada obtida na imagem.

\subsection{Características ópticas}

Foi realizada análise dos comprimentos de onda refletidos em determinado ângulo através de um espectrômetro (Figura 5). No arranjo montado foi utilizado um laser emissor de luz branca (A), um detector colimador (B) conectado ao espectrômetro por meio de uma fibra óptica e a amostra fixada em uma base com movimentos angulares (C). A amostra foi posicionada a um ângulo de $20^{\circ}$ em relação à normal do emissor de luz (ângulo de incidência) e o detector a um ângulo de $23^{\circ}$ em relação à linha paralela da face da amostra. Foram obtidas medidas na região cuja sobreposição $\mathrm{N}$ de pulsos foi de 2,5 e na região com $\mathrm{N}=6,25$ pulsos. 

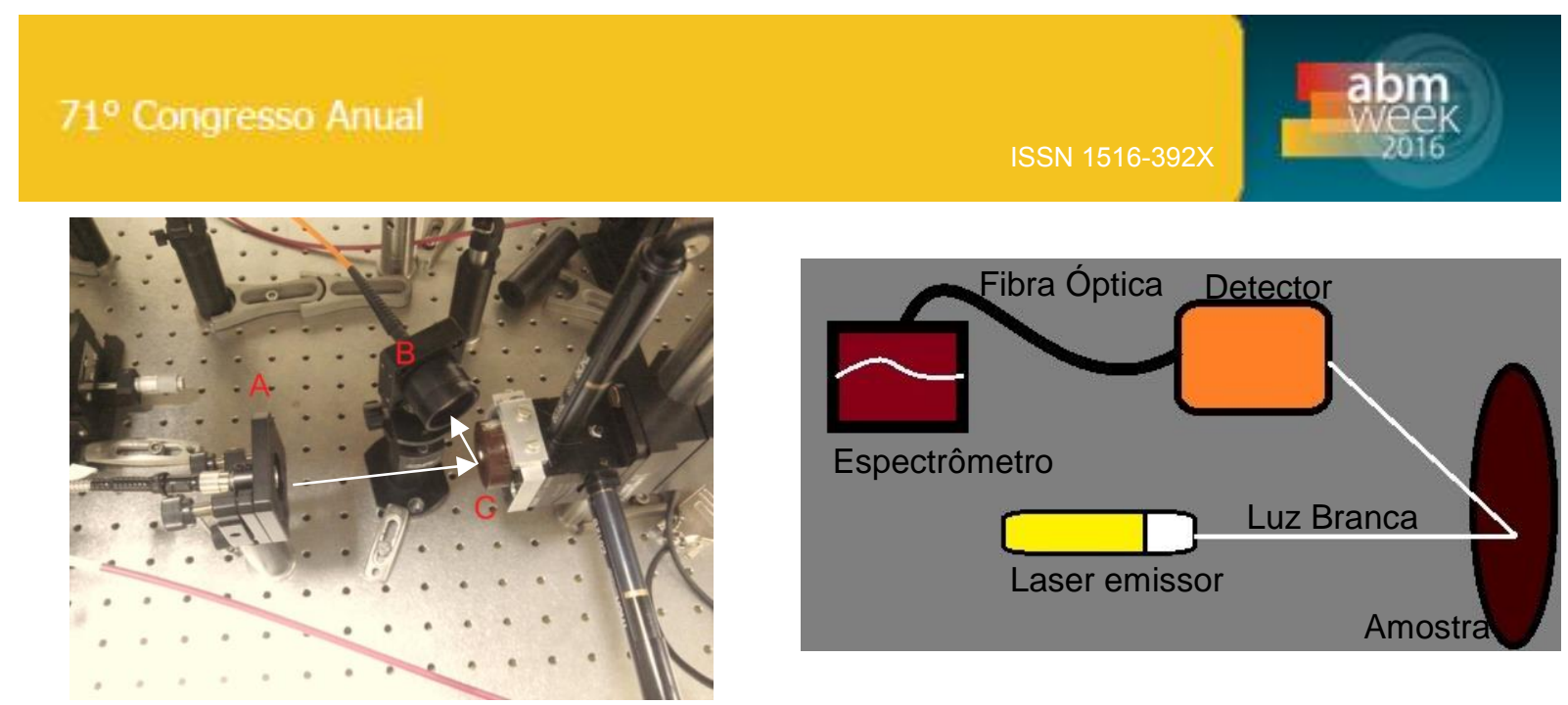

Figura 5. Arranjo para caracterização óptica

O resultado obtido após uma série de medidas está resumido no gráfico abaixo (figura 6), onde mostra que nas duas condições de sobreposição de pulsos houve um deslocamento do espectro emitido do refletido, quantificando o efeito visual de mudança de cor. É possível concluir também que, na condição com maior sobreposição de pulsos a intensidade da luz refletida foi em média $60 \%$ menor. Essa perda de intensidade pode ser justificada pela mudança nas características dos ripples, que conforme pode ser percebido na figura 2, há uma perda da característica de grade de difração e um início de formação de uma estrutura colunar, o que acarreta em uma maior absorção da luz incidida, causando o efeito de escurecimento da amostra.

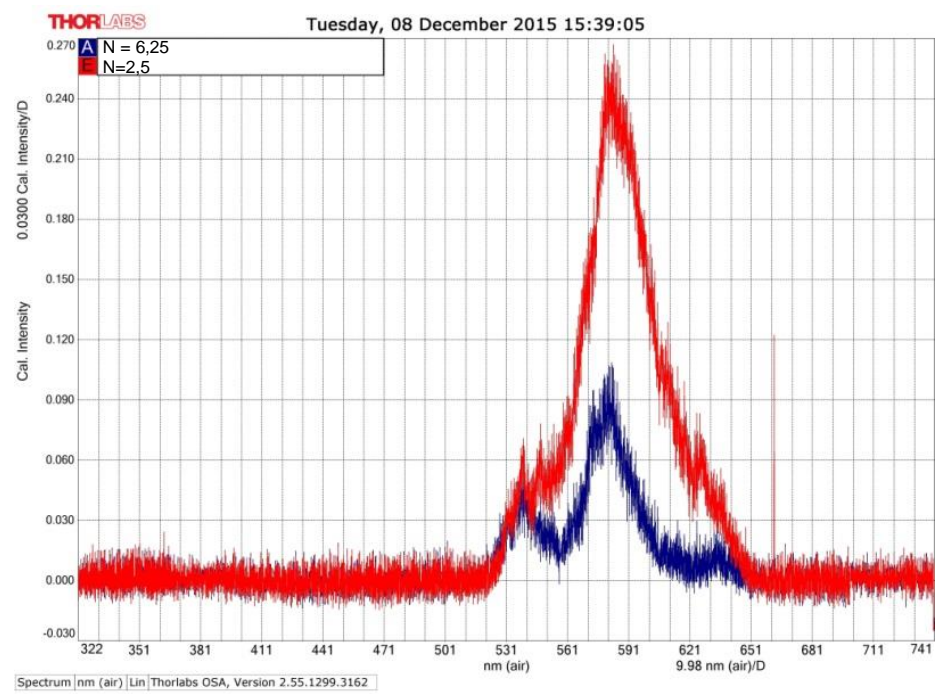

Figura 6. Comparação da emissão em função da taxa de sobreposição de pulsos

\section{CONCLUSÃO}

A partir da análise visual da amostra irradiada com laser de femtossegundo nas condições apresentadas acima, é possível comprovar que a formação das nanoestruturas periódicas causa uma alteração na coloração do metal. Entretanto, o aspecto visual não é alterado para uma cor fixa, e sim para um conjunto de cores correspondentes ao espectro de luz visível ao olho humano. Esse efeito ocorre pelo fato de as nanoestruturas periódicas atuarem como uma grade de difração na superfície do metal refletindo cores que são uma função do ângulo de incidência e do ângulo de visualização. Pode-se também observar que ao modificar as condições de irradiação, é possível criar uma superfície absorvedora de luz, ou seja, a 
superfície do metal fica com coloração preta, o que pode ser utilizado em diversas aplicações para marcação e detecção óptica.

\section{Agradecimentos}

Ao CNPq e à FAPESP, pelo auxílio financeiro através dos processos 134648/2015-3 e 2013/26113-6.

\section{REFERÊNCIAS}

1. Birnbaum M. Semiconductor Surface Damage Produced by Ruby Lasers. J Appl Phys. 1965;36(11):3688-9.

2. Huang M, Zhao F, Cheng $Y, X u N, X u$ Z. Origin of Laser-Induced Near-Subwavelength Ripples: Interference between Surface Plasmons and Incident Laser. ACS Nano [Internet]. 2009;3(12):4062-70. Available from: http://pubs.acs.org/doi/abs/10.1021/nn900654v

3. Yao J, Zhang C, Liu H, Dai Q, Wu L, Lan S, et al. Selective appearance of several laser-induced periodic surface structure patterns on a metal surface using structural colors produced by femtosecond laser pulses. Appl Surf Sci [Internet]. Elsevier B.V.; 2012;258(19):7625-32. Available from:

http://linkinghub.elsevier.com/retrieve/pii/S0169433212007593

4. Bonse J, Rosenfeld A, Krüger J. On the role of surface plasmon polaritons in the formation of laser-induced periodic surface structures upon irradiation of silicon by femtosecond-laser pulses. J Appl Phys [Internet]. 2009;106(10):104910. Available from: http://scitation.aip.org/content/aip/journal/jap/106/10/10.1063/1.3261734

5. Bonse J, Rosenfeld A, Krüger J. Implications of transient changes of optical and surface properties of solids during femtosecond laser pulse irradiation to the formation of laser-induced periodic surface structures. Appl Surf Sci [Internet]. Elsevier B.V.; 2011;257(12):5420-3. Available from: http://linkinghub.elsevier.com/retrieve/pii/S0169433210015825

6. Vorobyev AY, Guo C. Colorizing metals with femtosecond laser pulses. Appl Phys Lett [Internet]. 2008;92(4):041914. Available from: http://scitation.aip.org/content/aip/journal/apl/92/4/10.1063/1.2834902

7. Nolte S. Micromachining. In: Ferman ME, Galvanauskas A, Gregg Sucha, editors. Ultrafast Lasers: Technology and Applications. Marcel Denker; 2003. p. 359-95.

8. Oliveira V, Cunha A, Vilar R. Multi-scaled femtosecond laser structuring of stationary titanium surfaces. J Optoelectron Adv Mater. 2010;12(3):654-8. 